Pacific Journal of Mathematics

ON RESTRICTING IRREDUCIBLE CHARACTERS TO 


\title{
ON RESTRICTING IRREDUCIBLE CHARACTERS TO NORMAL SUBGROUPS
}

\author{
RICHARD L. ROTH
}

This paper is about the situation where $\chi$ is an irreducible character of a finite group $G$ and $K$ is a normal subgroup. A construction of Serre's relating the characters of $G$ with those of $G / K$ is used to give a new proof of a well-known lemma concerning the case that $\left.\chi\right|_{K}$ is irreducible and to generalize this lemma. It is seen that the irreducibility of $\left.\chi\right|_{K}$ is equivalent to the property that $(1 /|K|) \sum_{x \in h K}|\chi(x)|^{2}=1$ for each coset of $G$ modulo $K$ and also to the property that $\chi$ is not a component of $\lambda \chi$ for any irreducible character $\lambda$ of $G / K$ except for $\lambda=1$. The subgroup $J_{1}=J_{1}(\chi)$ is defined as the intersection of the kernels of the irreducible characters $\lambda$ of $G / K$ for which $\chi$ is a component of $\lambda \chi$. It is seen that an irreducible component $\sigma$ of the restriction of $\chi$ to $K$ will extend to $J_{1}, e_{J_{1}}(\chi)=e_{K}(\chi)$ and $J_{1}$ is the maximal normal subgroup with these two properties.

Preliminary remarks. $\hat{G}$ denotes the set of irreducible complex characters of $G$. 1 will often be used for the one-character of the appropriate group (according to context). $\langle\chi, \varphi\rangle_{G}=(1 /|G|) \sum_{g \in G} \chi(g) \bar{\varphi}(g)$, the usual inner product.

We include here a couple of well-known theorems to be referred to later.

THEOREM A. (Clifford) If $K \triangleleft G, \chi \in \hat{G}, \sigma \in \hat{K}$ and $\sigma$ a component of $\left.\chi\right|_{K}$ then $\left.\chi\right|_{K}=e_{K}(\chi) \sum_{i=1}^{m} \sigma^{g}$ where $e_{K}(\chi)$ is a positive integer called the ramification index, $m=[G: I(\sigma)]$ with $I(\sigma)$ being the inertial group for $\sigma$ and $\left\{g_{1}, \cdots, g_{m}\right\}$ are a set of coset representatives for $G$ modulo $I(\sigma)$. (See for example [1, Theorem 9.10].)

THEOREM B. Let $K \triangleleft G$ and $\chi$ an irreducible character of $G$ which remains irreducible when restricted to $K$. Then the characters $\lambda \chi$ are distinct and irreducible as $\lambda$ varies over the characters of $G / K$. Further if $\theta$ is an irreducible character of $G$ such that $\left.\chi\right|_{K}$ is a component of $\left.\theta\right|_{K}$, then $\theta$ is of the form $\lambda \chi$ as above. (See [3, Lemma 3.1].)

1. In this section we review a construction due to Serre which bears some resemblance to the familiar process for inducing characters from a subgroup. Theorem 1.1(b) is analogous to the Frobenius reciprocity theorem and was stated by Serre without proof in $[8$, p. 106]. 
By a class function on a group $G$ is meant any function from $G$ to the complex numbers which is constant on conjugacy classes. Let $K$ be a normal subgroup of the finite group $G$. If $\varphi$ is any class function of $G / K$, let $\varphi^{*}$ denote the corresponding class function on $G$ obtained in the usual way by $\varphi^{*}(g)=\varphi(g K)$. [Note that it is usually the custom to write $\varphi$ instead of $\varphi^{*}$ and this will be done in the latter part of this paper but here it is useful to make the distinction.] If $\psi$ is a class function on $G$ let $\psi *$ denote the function on $G / K$ defined by $\psi_{*}(h K)=(1 /|K|) \sum_{x \in h K} \psi(x)$.

THEOREM 1.1. (Serre) (a) $\psi_{*}$ is a class function on $G / K$. $G / K$.

(b) $\left\langle\varphi^{*}, \psi\right\rangle_{G}=\left\langle\varphi, \psi_{*}\right\rangle_{G \mid K}$ where $\varphi$ is any class function on

Proof. (a) If $h K$ and $h_{1} K$ are conjugate in $G / K$ then $h_{1} K=$ $g^{-1} h K g$ for some $g \in G$. Hence

$$
\begin{aligned}
\psi_{*}\left(h_{1} K\right) & =\frac{1}{|K|} \sum_{x \in h_{1} K} \psi(x)=\frac{1}{|K|} \sum_{x \in g^{-1} h K g} \psi(x) \\
& =\frac{1}{|K|} \sum_{y \in h K} \psi\left(g^{-1} y g\right)=\frac{1}{|K|} \sum_{y \in h K} \psi(y)=\psi_{*}(h K) .
\end{aligned}
$$

( b )

$$
\begin{aligned}
\left\langle\varphi, \psi_{*}\right\rangle_{G / K} & =\frac{1}{|G / K|} \sum_{h K \in G / K} \varphi(h K) \overline{\psi_{*}(h K)} \\
& =\frac{|K|}{|G|} \sum_{h K \in G / K}\left[\varphi(h K) \cdot \frac{1}{|K|} \sum_{x \in h K} \overline{\psi(x)}\right] \\
& =\frac{1}{|G|} \sum_{x \in G} \varphi^{*}(x) \overline{\psi(x)}=\left\langle\varphi^{*}, \psi\right\rangle_{G} .
\end{aligned}
$$

The following corollary shows that the construction appears not as promising as Frobenius' induction; nevertheless it has some use as will be seen shortly.

Corollary 1.2. Let $\psi \in \hat{G}$.

(a) If $K \subseteq \operatorname{Ker} \psi$ and hence $\psi$ may be regarded also as an element of $\widehat{G / K}$, then $\psi_{*}=\psi$ (under the latter identification).

(b) If $K \nsubseteq \operatorname{Ker} \psi$ then $\psi_{*} \equiv 0$.

Proof. If $\varphi \in \widehat{G / K}$ then $\left\langle\varphi, \psi_{*}\right\rangle_{G l K}=\left\langle\varphi^{*}, \psi\right\rangle_{G}=1$ or 0 depending on whether $\psi=\varphi^{*}$ or not. Case (b) means that $\psi \neq \varphi^{*}$ for any $\varphi \in \widehat{G / K}$ and since $\widehat{G / K}$ forms a basis for the class functions on $G / K$ we get that $\psi_{*} \equiv 0$. If (a) holds, then $\psi=\varphi^{*}$ for exactly one $\varphi$ and so $\psi_{*}=\varphi$.

COROLlaRY 1.3. If $\psi$ is any class function on $G$ write $\psi=$ 
$\sum a_{i} \chi_{i}+\sum b_{j} \psi_{j}$ where $\chi_{i}, \psi_{j} \in \hat{G}, K \subseteq \operatorname{Ker} \chi_{i}$ each $i$ but $K \nsubseteq \operatorname{Ker} \psi_{j}$ each $j$. Then $\left(\psi_{*}\right)^{*}=\sum a_{i} \chi_{i}$. Further if $\psi$ is a character then $\left(\psi_{*}\right)^{*}$ is also a character or the zero function.

In what follows, we omit the upper star and identify characters of $G / K$ with characters of $G$.

2. We now use the Serre construction to give a proof of a theorem which generalizes both [2, Lemma, p. 178] of Gallagher and [5, Lemma 4.2] of Iwahori and Matsumoto (see corollaries which follow).

THEOREM 2.1. Let $\chi \in \hat{G}$. Let $S(\chi)$ denote the set of irreducible characters $\lambda$ of $G$ such that $\lambda \chi$ contains $\chi$ as a component, i.e., $\langle\lambda \chi, \chi\rangle_{G}=n_{\lambda}>0$. Then $(\chi \bar{\chi})_{*}=\sum_{\lambda \in S(\chi) \cap \widehat{G \mid K}} n_{\lambda} \lambda$ i.e., $(1 /|K|) \sum_{x \in h K}|\chi(x)|^{2}=$ $\sum \sum_{i \in S(x) \cap \widehat{G \mid K}} n_{\lambda} \lambda(h K)$.

Proof. $n_{\lambda}=\langle\lambda \chi, \chi\rangle_{G}=\langle\lambda, \chi \bar{\chi}\rangle_{G}$ so that $\chi \bar{\chi}=\sum n_{\lambda} \lambda$ summed over $\lambda \in S(\chi)$. By Corollary 1.3, $(\chi \bar{\chi})_{*}=\sum_{\lambda \in S(\chi) \cap \widehat{G \mid K}} n_{\lambda} \lambda$.

COROLlaRY 2.2. The one-character always occurs with multiplicity one in $(\chi \bar{\chi})_{*}$.

CoRollary 2.3. (Iwahori-Matsumoto [5, Lemma 4.2]) If $G / K$ is abelian and $H(\chi)$ is the group of (linear) characters $\lambda \in \widehat{G / K}$ such that $\lambda \chi=\chi$ then $(\chi \bar{\chi})_{*}=\sum_{\lambda \in H(x)} \lambda$.

Proof. In this case $S(\chi) \cap \widehat{G / K}=H(\chi)$ since if $\lambda$ is linear and $\langle\lambda \chi, \chi\rangle=n_{\lambda}>0$ then $\lambda \chi=\chi$ and $n_{\lambda}=1$.

CoRollary 2.4. (Gallagher [2, Lemma, p. 178]; also Isaacs [4, Lemma 3.4]) If $\left.\chi\right|_{k}$ is irreducible then $(\chi \bar{\chi})_{*}=1$.

It is instructive to give two different short proofs.

Proof 1. By Theorem B in the preliminary remarks the characters $\{\lambda \chi: \lambda \in \widehat{G / N}\}$ are all distinct and irreducible. Thus $S(\chi) \cap \widehat{G / N}=$ $\{1\}$.

Proof 2. $\left.\chi\right|_{K}$ irreducible means that

$$
1=\langle\chi, \chi\rangle_{K}=\frac{1}{|K|} \sum \chi(g) \overline{\chi(g)}=(\chi \bar{\chi})_{*}(K) .
$$

Hence $(\chi \bar{\chi})_{*}$ is a character (Corollary 1.3) of degree 1. By Corollary 
2.2, we have $(\chi \bar{\chi})_{*}=1$.

CoRollary 2.5 (the converse to Corollary 2.4). If $(\chi \bar{\chi})_{*}=1$ then $\left.\chi\right|_{K}$ is irreducible.

Proof. As in Proof 2 of Corollary 2.4 above, note that $\langle\chi, \chi\rangle_{K}=$ $(\chi \bar{\chi})_{*}(K)=1$.

As a summary it is convenient to make a list of equivalent statements.

THEOREM 2.5. Let $\chi \in \hat{G}, K \triangleleft G$. The following conditions are equivalent:

(a) $\left.\chi\right|_{K}$ is irreducible.

(b) $(\chi \bar{\chi})_{*}=1$.

(c) If $\lambda \in \widehat{G / K}$ and $\langle\lambda \chi, \chi\rangle_{G} \neq 0$ then $\lambda=1$.

(d) The characters in the set $\{\lambda \chi: \lambda \in \widehat{G / K}\}$ are distinct and irreducible.

Proof. (a) $\Leftrightarrow$ (b) by Corollaries 2.4 and 2.5. $\quad(b) \Leftrightarrow(c)$ by Theorem 2.1. So (a), (b) and (c) are equivalent. Clearly (d) $\Rightarrow(\mathrm{c}) . \quad(\mathrm{a}) \Rightarrow(\mathrm{d})$ is by Theorem $\mathrm{B}$ of the preliminary remarks.

3. In [6] the author considered the effect of the characters $\widehat{G / K}$ on an irreducible character of $G$ in the case that $G / K$ is abelian (see also [5] for a similar treatment). In particular the irreducible characters $\mathrm{H}(\chi)$ that "fix" $\chi$ (i.e., $\lambda \chi=\chi$ ) were studied and the intersection of their kernels was singled out as the "dual inertial group" $J(\chi)$. If $G / K$ is non-abelian then its irreducible characters need not be linear, and there are several ways to generalize the above concept. In [7] we called $H(\chi)$ the set of irreducible characters $\lambda$ such that $\lambda \chi=(\operatorname{deg} \lambda) \chi$. Some properties of $J(\chi)$ were dealt with there where $J(\chi)$ is the intersection of the kernels of set of characters $H(\chi)$. An alternative approach which we look at briefly here is to examine instead $H_{1}(\chi)=S(\chi) \cap \widehat{G / K}=$ the irreducible characters $\lambda$ of $G / K$ such that $\lambda \chi$ contains $\chi$ as a component. Then let $J_{1}(\chi)=$ $\bigcap\left\{\operatorname{Ker} \lambda: \lambda \in H_{1}(\chi)\right\}$. It is seen below that $J_{1}=J_{1}(\chi)$ has at least some of the properties of the "dual inertial group" of [6], namely that if, (1) $\sigma$ is a component of $\left.\chi\right|_{K}$ then $\sigma$ may be extended to $J_{1}(\chi)$ and $(2) e_{J_{1}}(\chi)=e_{K}(\chi)$. Further it is shown (Theorem 3.5) that $J_{1}(\chi)$ might be characterized as the (unique) maximal normal subgroup between $G$ and $K$ having these two properties. (This latter fact is new even for the case of $G / K$ abelian treated in [6].) 
TheOREM 3.1. Let $\chi \in \hat{G}, K \triangleleft G$, and $J_{1}=J_{1}(\chi)$ be defined as above. Let $\psi$ be an irreducible component of $\left.\chi\right|_{J_{1}}$. Then $(\psi \bar{\psi})_{*}=1$ on $J_{1} / K$ and hence $\left.\psi\right|_{K}$ is irreducible.

Proof. $(\chi \bar{\chi})_{*}=\sum_{\lambda \in H_{1}(\chi)} n_{\lambda} \lambda$. So $(\chi \bar{\chi})_{*}$ restricted to $J_{1} / K$ consists of a multiple of the one-character. Since $\psi$ is a component of $\left.\chi\right|_{J_{1}}$, $\left.\chi \bar{\chi}\right|_{J_{1}}=\psi \bar{\psi}+\tau$ where $\tau$ is another character of $J_{1}$, and the restriction of $(\chi \bar{\chi})_{*}$ to $J_{1} / K$ equals $(\psi \bar{\psi})_{*}+\tau_{*}$. Hence $(\psi \bar{\psi})_{*}$ is a multiple of the one-character, and hence is the one-character by Corollary 2.2.

CoROLLARY 3.2. Let $K \triangleleft G, \chi \in \hat{G}$ and let $\sigma$ be a component of $\left.\chi\right|_{K}$. Then $\sigma$ may be extended to a character $\psi$ of $J_{1}$ and $I(\sigma) \supseteqq J_{1}(\chi)$ where $I(\sigma)$ denotes the inertial group of $\sigma$.

Proof. Let $\psi$ be a component of $\left.\chi\right|_{K}$. By Theorem $\left.3.1 \psi\right|_{K}=\tau$ is an irreducible component of $\left.\chi\right|_{K}$. For some $g \in G, \sigma=\tau^{g}$ (by Theorem A in the preliminary remarks) and $\psi^{g}$ is an extension of $\sigma$ to $J_{1}$. For simplicity of notation we may assume henceforth that $\left.\psi\right|_{K}=\sigma$. Thus if $h \in J_{1}$ then $\psi^{h}=\psi$ so $\sigma^{h}=\sigma$ and hence $h \in I(\sigma)$.

The following notation which was used in [7] will be helpful in proving Theorem 3.3. If $\rho, \chi \in G$ then $\rho^{*} \chi$ is the set of irreducible components of $\rho \chi$. If $T \leqq \hat{G}$ then $\rho^{*} T=\bigcup\left\{\rho^{*} \gamma \mid \gamma \in T\right\}=T^{*} \rho$. Associativity holds: $\left(\rho^{*} \gamma\right) * \tau=\rho^{*}\left(\gamma^{*} \tau\right)=$ the irreducible components of $\rho \gamma \tau$. In this notation, $S(\chi)=\chi^{*} \bar{\chi}$ since $\langle\lambda \chi, \chi\rangle=\langle\lambda, \chi \bar{\chi}\rangle$. Theorem 2.5 of [7] states that if $K \triangleleft G . \quad \chi \in \hat{G}$ and $\psi$ an irreducible component of $\left.\chi\right|_{K}$ then the set of irreducible components of $\psi^{G}$ equals $\mathrm{U}\left\{\chi^{*} \rho \mid \rho \in \widehat{G / K}\right\}=\chi^{*} \widehat{G / K}$.

THEOREM 3.3. $e_{K}(\chi)=e_{J_{1}}(\chi)$.

Proof. Let $\psi$ be an irreducible component of $\left.\chi\right|_{J_{1}}$ and $\left.\psi\right|_{K}=\sigma$. then

$$
\left.\chi\right|_{K}=e_{K}(\chi) \sum_{i=1}^{m} \sigma^{g_{i}} \quad m=[G: I(\sigma)]
$$

and

$$
\left.\chi\right|_{J_{1}}=e_{J_{1}}(\psi) \sum_{i=1}^{n} \psi^{h_{i}} \quad n=[G: I(\psi)]
$$

by Clifford's theorem ("Theorem A"). Since $\left.\psi\right|_{K}=\sigma$ it is clear that $I(\psi) \subseteq I(\sigma)$. It suffices to prove that $I(\psi)=I(\sigma)$ for then $m=n$ and since $\operatorname{deg} \sigma=\operatorname{deg} \psi$ the above equations show that $e_{K}(\chi)=e_{J_{1}}(\chi)$. 
Hence let $g \in I(\sigma)$. We must show that $\psi^{g}=\psi$. Clearly $\left.\psi^{g}\right|_{K}=$ $\left.\psi\right|_{K}=\sigma$ hence by Theorem B, $\psi^{g}=\lambda \psi$ for some $\lambda \in \widehat{J_{1} / K}$. And $(\lambda \psi)^{G}=\left(\psi^{g}\right)^{G}=\psi^{G}$. Let $\gamma$ be an irreducible component of $\lambda^{G}$. Then $\left.\gamma\right|_{J_{1}}=e_{J_{1}}(\gamma) \sum \lambda^{g}$ and $K \cong \operatorname{Ker} \gamma$. Thus any irreducible component of $\psi^{\theta}=(\lambda \psi)^{G}$ must be included among the irreducible components of $\gamma \psi^{\theta}=\left(\left.\gamma\right|_{J_{1}} \psi\right)^{G}$. In particular, $\left\langle\chi, \psi^{G}\right\rangle>0$ and hence $\left\langle\chi, \gamma \psi^{G}\right\rangle>0$. By Theorem 2.5 of [7], cited earlier, there exists $\tau \in \widehat{G / J_{1}}$ such that $\chi \in \gamma^{*}\left(\tau^{*} \chi\right)=\left(\gamma^{*} \tau\right)^{*} \chi$. Hence there exists $\delta \in \gamma^{*} \tau$ such that $\chi \in \delta^{*} \chi$. Hence $\delta \in S(\chi)$.

Now $\gamma \in \widehat{G / K}, \quad \tau \in \widehat{G / J_{1}} \subseteq G / K$ so $\delta \in \gamma^{*} \tau \subseteq \widehat{G / K}$; i.e., $\delta \in S(\chi) \cap$ $\widehat{G / K}=H_{1}(\chi)$. Thus $J_{1}(\chi) \subseteq \operatorname{Ker} \delta$; i.e., $\delta \in \widehat{G / J_{1}}$. But $\langle\delta, \gamma \tau\rangle>0$ means that $\langle\delta \bar{\tau}, \gamma\rangle>0$ so $\gamma \in \delta^{*} \bar{\tau} \leqq \widehat{G / J}_{1}$ so that $J_{1} \subseteq \operatorname{Ker} \gamma$ and hence $\lambda$ is trivial. Thus $\psi^{g}=\lambda \psi=\psi$.

CoROLlaRY 3.4. $I(\psi)=I(\sigma)$.

TheOREM 3.5. Let G, $K, \chi, \sigma$ be as in the previous theorems. Let $N$ be a normal subgroup of $G$ containing $K$ such that

(1) $e_{N}(\chi)=e_{K}(\chi)$ and

(2) $\sigma$ extends to an irreducible character $\theta$ of $N$. Then $N \subseteq$ $J_{1}(\chi)$. Hence $J_{1}(\chi)$ is the unique normal subgroup which is maximal with respect to having properties (1) and (2).

Proof. Since $\left.\theta\right|_{K}=\sigma$ is irreducible, $\left.\theta^{h}\right|_{K}=\sigma^{h}$ is irreducible for each $h \in G$. Using Theorem A and writing $e=e_{K}(\chi)=e_{N}(\chi)$ we have:

$$
\begin{array}{ll}
\chi_{K}=e \sum \sigma^{h} & \left\{\sigma^{h}\right\}=\text { set of distinct conjugates of } \sigma, \\
\left.\chi\right|_{N}=e \sum \theta^{g} & \left\{\theta^{g}\right\}=\text { set of distinct conjugates of } \theta .
\end{array}
$$

Since $\left.\chi\right|_{K}=\left.\left(\left.\chi\right|_{N}\right)\right|_{K}$ we see that different conjugates $\theta^{h}$ of $\theta$ must restrict to different conjugates of $\sigma$. Hence if $\theta \neq \theta^{h}$ then $\sigma=$ $\left.\theta\right|_{K} \neq\left.\theta^{h}\right|_{K}=\sigma^{h}$.

Now let $\gamma \in H_{1}(\chi)$. We will show that $N \leqq \operatorname{Ker} \gamma$ and hence $N \cong J_{1}(\chi)=\bigcap\left\{\operatorname{Ker} \gamma: \gamma \in H_{1}(\chi)\right\}$.

$\gamma \in H_{1}(\chi)$ means $\chi \in \gamma^{*} \chi$ and hence $\left.\left.\gamma\right|_{N} \chi\right|_{N}$ contains $\theta$ as a component. Hence there exists $\lambda$ an irreducible component of $\left.\gamma\right|_{N}$, and $\theta^{g}$ a conjugate of $\theta$ such that $\theta \in \lambda^{*} \theta^{g}$. So $\left.\left(\lambda \theta^{g}\right)\right|_{N}$ contains $\sigma$ as a component and $\left.\operatorname{yet}\left(\lambda \theta^{g}\right)\right|_{N}=(\operatorname{deg} \lambda) \sigma^{g}$. Thus $\sigma=\sigma^{g}$ and by the initial discussion this means that $\theta=\theta^{g}$ and that $\theta \in \lambda^{*} \theta$. By Theorem $B, \lambda$ must be trivial. Since any component of $\left.\gamma\right|_{N}$ is a conjugate of $\lambda$, we have that $N \leqq \operatorname{Ker} \gamma$. 


\section{REFERENCES}

1. Walter Feit, Characters of finite groups (Benjamin, New York, 1967).

2. P.X. Gallagher, The number of conjugacy classes in a finite group, Math. Z., 118 (1970), 175-179.

3. I. M. Isaacs, Extensions of certain linear groups, J. Algebra, 4 (1966), 3-12.

4. - Characters of solvable and symplectic groups, Amer. J. Math., 95 (1973), 594-635.

5. N. Iwahori and H. Matsumoto, Several remarks on projective representations of finite groups, Jr. Fac. Sci. Univ. Tokyo, Section 2, Math. Astro. Phys. Chem., 10 (1964), 129-146.

6. R.L. Roth, $A$ dual view of the Clifford theory of the characters of finite groups, Canad. J. Math., 23 (1971), 857-865.

7. - Character and conjugacy class hypergroups of a finite group, Ann. di Mat. Pura ed Aplicata. To appear.

8. J. Serre, Corps Locaux, Actualites Scientifiques et Industrielles, No. 1296, Hermann, 1962.

Received December 26, 1974.

UNiversity of Colorado, Boulder, Colorado 



\section{PACIFIC JOURNAL OF MATHEMATICS}

\section{EDITORS}

RICHARD ARENS (Managing Editor) University of California

Los Angeles, California 90024

\section{R. A. Beaumont}

University of Washington Seattle, Washington 98105
J. DugundJI Department of Mathematics University of Southern Californıa Los Angeles, California 90007

D. Gilbarg aNd J. Milgram Stanford University Stanford, California 94305

\section{ASSOCIATE EDITORS}

E. F. BeCKENBACH

B. H. NeUmanN

F. Wolf

K. YoSHIDA

\section{SUPPORTING INSTITUTIONS}

UNIVERSITY OF BRITISH COLUMBIA CALIFORNIA INSTITUTE OF TECHNOLOGY UNIVERSITY OF CALIFORNIA MONTANA STATE UNIVERSITY UNIVERSITY OF NEVADA NEW MEXICO STATE UNIVERSITY OREGON STATE UNIVERSITY UNIVERSITY OF OREGON OSAKA UNIVERSITY
UNIVERSITY OF SOUTHERN CALIFORNIA STANFORD UNIVERSITY UNIVERSITY OF TOKYO UNIVERSITY OF UTAH WASHINGTON STATE UNIVERSITY UNIVERSITY OF WASHINGTON AMERICAN MATHEMATICAL SOCIETY NAVAL WEAPONS CENTER 


\section{Pacific Journal of Mathematics \\ Vol. 59, No. 1 \\ May, 1975}

Shashi Prabha Arya and M. K. Singal, More sum theorems for topological

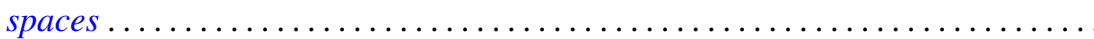

Goro Azumaya, F. Mbuntum and Kalathoor Varadarajan, On M-projective and

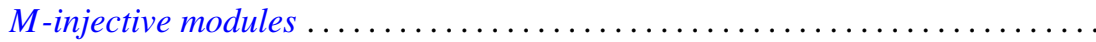

Kong Ming Chong, Spectral inequalities involving the infima and suprema of

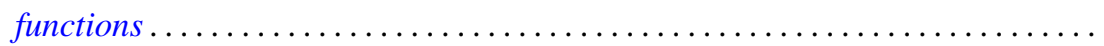

Alan Hetherington Durfee, The characteristic polynomial of the monodromy .......

Emilio Gagliardo and Clifford Alfons Kottman, Fixed points for orientation

preserving homeomorphisms of the plane which interchange two points ......

Raymond F. Gittings, Finite-to-one open maps of generalized metric spaces .......

Andrew M. W. Glass, W. Charles (Wilbur) Holland Jr. and Stephen H. McCleary,

$a^{*}$-closures of completely distributive lattice-ordered groups .............

Matthew Gould, Endomorphism and automorphism structure of direct squares of

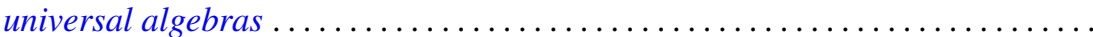

R. E. Harrell and Les Andrew Karlovitz, On tree structures in Banach spaces .....

Julien O. Hennefeld, Finding a maximal subalgebra on which the two Arens

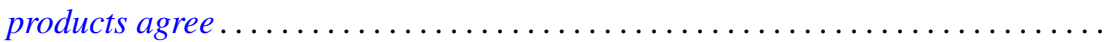

William Francis Keigher, Adjunctions and comonads in differential algebra .......

Robert Bernard Kelman, A Dirichlet-Jordan theorem for dual trigonometric

series

Allan Morton Krall, Stieltjes differential-boundary operators. III. Multivalued operators-linear relations...

Hui-Hsiung Kuo, On Gross differentiation on Banach spaces .

Tom Louton, A theorem on simultaneous observability ...

Kenneth Mandelberg, Amitsur cohomology for certain extensions of rings of algebraic integers.

Coy Lewis May, Automorphisms of compact Klein surfaces with boundary . . .

Peter A. McCoy, Generalized axisymmetric elliptic functions .

211

Muril Lynn Robertson, Concerning Siu's method for solving $y^{\prime}(t)=F(t$, $y(g(t)))$. .

Richard Lewis Roth, On restricting irreducible characters to normal

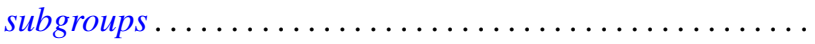

Albert Oscar Shar, $P$-primary decomposition of maps into an $H$-space .

Kenneth Barry Stolarsky, The sum of the distances to certain pointsets on the unit circle.

Bert Alan Taylor, Components of zero sets of analytic functions in $C^{2}$ in the unit ball or polydisc

Michel Valadier, Convex integrands on Souslin locally convex spaces ...

Januario Varela, Fields of automorphisms and derivations of $C$

Arnold Lewis Villone, A class of symmetric differential operators with deficiency

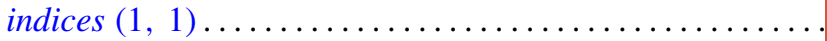

